\title{
An Out Space Accelerating Algorithm for Generalized Affine Multiplicative Programs Problem
}

\author{
Lei Cai, ${ }^{1}$ Shuai Tang, ${ }^{2}$ Jingben Yin, ${ }^{3}$ Zhisong Hou, ${ }^{1}$ and Hongwei Jiao ${ }^{3}$ \\ ${ }^{1}$ School of Information Engineering, Henan Institute of Science and Technology, Xinxiang 453003, China \\ ${ }^{2}$ Jiyuan Vocational and Technical College, Jiyuan 459000, China \\ ${ }^{3}$ School of Mathematical Sciences, Henan Institute of Science and Technology, Xinxiang 453003, China \\ Correspondence should be addressed to Lei Cai; cailei2014@126.com and Jingben Yin; jingbenyin@163.com
}

Received 18 February 2017; Accepted 4 May 2017; Published 14 June 2017

Academic Editor: Qiang Song

Copyright (c) 2017 Lei Cai et al. This is an open access article distributed under the Creative Commons Attribution License, which permits unrestricted use, distribution, and reproduction in any medium, provided the original work is properly cited.

\begin{abstract}
This paper presents an out space branch-and-bound algorithm for solving generalized affine multiplicative programs problem. Firstly, by introducing new variables and constraints, we transform the original problem into an equivalent nonconvex programs problem. Secondly, by utilizing new linear relaxation technique, we establish the linear relaxation programs problem of the equivalent problem. Thirdly, based on the out space partition and the linear relaxation programs problem, we construct an out space branch-and-bound algorithm. Fourthly, to improve the computational efficiency of the algorithm, an out space reduction operation is employed as an accelerating device for deleting a large part of the investigated out space region. Finally, the global convergence of the algorithm is proved, and numerical results demonstrate the feasibility and effectiveness of the proposed algorithm.
\end{abstract}

\section{Introduction}

The generalized affine multiplicative programs problem (GAMP) arises naturally in many practical applications including management science, engineering optimization design, optimal control, Euclidean geometry, economic planning, production planning, and combinatorial mathematics $[1,2]$. The problem (GAMP) usually contains many local optimum points which are not global optimum solution, so that important theoretical difficulties and computational complexities exist. Therefore, it is very necessary to establish an effective algorithm for globally solving the problem (GAMP).

The mathematical modelling of the generalized affine multiplicative programs problem is given as follows:

$$
\begin{aligned}
v=\max \quad f(y) & =\sum_{i=1}^{p}\left(\sum_{j=1}^{n} c_{i j} y_{j}+d_{i}\right)\left(\sum_{j=1}^{n} e_{i j} y_{j}+f_{i}\right) \\
\text { s.t. } \quad y \in Y & =\{y \mid A y \leq b\},
\end{aligned}
$$

where $p \geq 2, c_{i j}, e_{i j}, d_{i}, f_{i} \in R, i=1, \ldots, p, j=1, \ldots, n$.

In the last decades, many algorithms have been intended for solving the generalized linear multiplicative programming problem, such as parametric simplex algorithms $[3,4]$, quadratic programming algorithms [5-9], rectangle branchand-bound algorithms $[10,11]$, approximate algorithms [12, 13], finite algorithm [14], outcome space algorithm [15], cutting plane algorithm [16], heuristic algorithm [17], monotonic 
optimization algorithms [18, 19], and simplicial branch-andbound algorithm [20]. Although there exist some algorithms which can be used to solve the generalized linear multiplicative programming problem, as far as we know, it is still necessary to propose a more efficient algorithm for globally solving (GAMP).

In this paper, we shall present an effective algorithm for globally solving (GAMP). First of all, we transform (GAMP) into an equivalent problem (EQ). Secondly, by utilizing the new linear relaxation technique, we systematically convert (EQ) into a series of linear relaxation programs problems, which can infinitely approximate the global optimal solution of (EQ) by a successive partition. Thirdly, based on the out space partition and the linear relaxation programs problem, we construct an out space branch-and-bound algorithm. In this algorithm, the proposed branching operation takes place in $R^{p}$, rather than $R^{n}$ or $R^{2 p}$; this economizes the required computation. Fourthly, to improve the computational efficiency of the algorithm, an out space reduction operation is employed as an accelerating device for deleting a large part of the investigated out space region. Finally, the global convergence of the algorithm is proved, and numerical experimental results demonstrate the feasibility and effectiveness of the proposed algorithm.

This paper is organized as follows. In Section 2, by introducing new variables and constraints, we establish the equivalent problem (EQ) of the initial problem (GAMP). Next, by utilizing the linearization technique, we derive the linear relaxation programming problem of (EQ), which can provide a reliable upper bound for the global optimal value of the (EQ). In Section 3, an out space region reduction operation is introduced, and by combining this operation with bounding technique, an out space accelerating branch-andbound algorithm is established and its global convergence is proved. Section 4 presents the numerical experimental results obtained by solving some test examples. Finally, the some concluding remarks of this paper are elaborated.

\section{Equivalent Problem and Its Linear Relaxation}

In this section, we will convert the problem (GAMP) into an equivalent nonconvex problem (EQ). Since $\sum_{j=1}^{n} c_{i j} y_{j}+d_{i}$ and $\sum_{j=1}^{n} e_{i j} y_{j}+f_{i}$ are all finite affine functions defined in $Y$, there exist positive constant numbers $M_{i}$ and $N_{i}$ such that $\sum_{j=1}^{n} c_{i j} y_{j}+d_{i}+M_{i}>0$ and $\sum_{j=1}^{n} e_{i j} y_{j}+f_{i}+N_{i}>0$ for any $y \in Y$. Therefore, in the following, without loss of generality, we can assume that $\sum_{j=1}^{n} c_{i j} y_{j}+d_{i} \geq 0$ and $\sum_{j=1}^{n} e_{i j} y_{j}+f_{i} \geq 0$ for all $y \in Y$.

Without loss of generality, we let

$$
\begin{aligned}
L_{i}^{0} & =\min _{y \in Y}\left(\sum_{j=1}^{n} e_{i j} y_{j}+f_{i}\right), \\
U_{i}^{0} & =\max _{y \in Y}\left(\sum_{j=1}^{n} e_{i j} y_{j}+f_{i}\right),
\end{aligned}
$$

Define the set $Z^{0}=\left\{z \in R^{p} \mid L_{i}^{0} \leq z_{i} \leq U_{i}^{0}, i=1, \ldots, p\right\}$. For any $y \in Y$ and $z \in Z^{0}$, define the function $g(y, z)=$ $\sum_{i=1}^{p}\left[z_{i}\left(\sum_{j=1}^{n} c_{i j} y_{j}+d_{i}\right)\right]$, and consider the following equivalent problem:

$$
\begin{array}{ll}
\max & g(y, z)=\sum_{i=1}^{p}\left[z_{i}\left(\sum_{j=1}^{n} c_{i j} y_{j}+d_{i}\right)\right] \\
\text { s.t. } & \sum_{j=1}^{n} e_{i j} y_{j}+f_{i}-z_{i} \geq 0, \quad i=1, \ldots, p, \\
& y \in Y \\
& z \in Z^{0} .
\end{array}
$$

Obviously, if $\left(y^{*}, z^{*}\right)$ is a global optimal solution for (EQ), then $y^{*}$ is a global optimal solution for (GAMP), and $z_{i}^{*}=$ $\sum_{j=1}^{n} e_{i j} y_{j}^{*}+f_{i}, i=1, \ldots, p$. If $y^{*}$ is a global optimal solution for (GAMP), then $\left(y^{*}, z^{*}\right)$ is a global optimal solution for (EQ), where $z_{i}^{*}=\sum_{j=1}^{n} e_{i j} y_{j}^{*}+f_{i}, i=1, \ldots, p$.

In the following, the main computation is to globally solve (EQ). Next, we will construct the linear relaxation programs problem of (EQ), which can provide the upper bounds for (EQ) in the proposed branch-and-bound algorithm. Assume that $Z=\left\{z \in R^{p} \mid L_{i} \leq z_{i} \leq U_{i}, i=1, \ldots, p\right\} \subseteq Z^{0}$. And without loss of generality, let

$$
\begin{aligned}
& T_{i}^{+}=\left\{j \mid c_{i j} \geq 0, j=1, \ldots, n\right\}, \\
& T_{i}^{-}=\left\{j \mid c_{i j}<0, j=1, \ldots, n\right\}, \\
& D_{i}^{+}=\left\{i \mid d_{i} \geq 0, i=1, \ldots, p\right\}, \\
& D_{i}^{-}=\left\{i \mid d_{i}<0, \quad i=1, \ldots, p\right\} .
\end{aligned}
$$

Then the linear relaxation programs $\left(U_{Z}\right)$ of $(\mathrm{EQ})$ in $Z$ can be given as follows:

$$
\begin{aligned}
u_{Z}=\max \sum_{i=1}^{p} & \left(\sum_{T_{i}^{+}} U_{i} c_{i j} y_{j}+\sum_{T_{i}^{-}} L_{i} c_{i j} y_{j}\right)+\sum_{D_{i}^{+}} U_{i} d_{i} \\
& +\sum_{D_{i}^{-}} L_{i} d_{i} \\
\text { s.t. } \quad L_{i} & -\left(\sum_{j=1}^{n} e_{i j} y_{j}+f_{i}\right) \leq 0, \quad i=1, \ldots, p, \\
& y \in Y,
\end{aligned}
$$

where (EQ) in $Z$ is defined by

$$
\begin{gathered}
v_{Z}=\max \quad g(y, z)=\sum_{i=1}^{p} z_{i}\left(\sum_{j=1}^{n} c_{i j} y_{j}+d_{i}\right) \\
\text { s.t. }\left(\sum_{j=1}^{n} e_{i j} y_{j}+f_{i}\right)-z_{i} \geq 0,
\end{gathered}
$$

$$
i=1, \ldots, p,
$$

$y \in Y$, $z \in Z \subseteq Z^{0}$. 
Theorem 1. (i) For any $Z \subseteq Z^{0}$, one has $u_{Z} \geq v_{Z}$. (ii) If $\bar{Z}=$ $\left\{z \in R^{p} \mid \bar{L} \leq z \leq \bar{U}\right\} \subseteq Z$ and $\bar{Z} \neq \emptyset$, then $u_{\bar{Z}} \leq u_{Z}$.

Proof. (i) If $v_{Z}=-\infty$, then we can easily obtain $u_{Z}=-\infty$; it is obvious that $u_{Z} \geq v_{Z}$, if $v_{Z}$ is finite and achieved. Denote by $(\bar{y}, \bar{z})$ a global optimal solution for $\left(\mathrm{EQ}_{Z}\right)$. Then $\bar{y} \in Y, \bar{z} \in Z$. For each $i \in\{1, \ldots, p\}$, we have $L_{i} \leq \bar{z}_{i} \leq U_{i}$. Therefore, we have

$$
\begin{aligned}
\sum_{i=1}^{p} \bar{z}_{i}\left(\sum_{j=1}^{n} c_{i j} \bar{y}_{j}+d_{i}\right) \leq & \sum_{i=1}^{p}\left(\sum_{T_{i}^{+}} U_{i} c_{i j} \bar{y}_{j}+\sum_{T_{i}^{-}} L_{i} c_{i j} \bar{y}_{j}\right) \\
& +\sum_{D_{i}^{+}} U_{i} d_{i}+\sum_{D_{i}^{-}} L_{i} d_{i} .
\end{aligned}
$$

Since $(\bar{y}, \bar{z})$ is a global optimal solution for $\left(\mathrm{EQ}_{Z}\right)$, it follows that

$$
v_{Z}=\sum_{i=1}^{p} \bar{z}_{i}\left(\sum_{j=1}^{n} c_{i j} \bar{y}_{j}+d_{i}\right)
$$

Since $\bar{y}$ is a feasible solution for $\left(U_{Z}\right)$,

$$
u_{Z} \geq \sum_{i=1}^{p}\left(\sum_{T_{i}^{+}} U_{i} c_{i j} \bar{y}_{j}+\sum_{T_{i}^{-}} L_{i} c_{i j} \bar{y}_{j}\right)+\sum_{D_{i}^{+}} U_{i} d_{i}+\sum_{D_{i}^{-}} L_{i} d_{i} .
$$

By the above discussions, we have $u_{Z} \geq v_{Z}$.

(ii) Let $\bar{Z}=\left\{z \in R^{p} \mid \bar{L} \leq z \leq \bar{U}\right\} \subseteq Z$ and $\bar{Z} \neq \emptyset$; we have the following conclusions.

If $\left(U_{Z}\right)$ is infeasible, then we can easily prove that $\left(U_{\bar{Z}}\right)$ is also infeasible; the conclusion is obvious.

If $\left(U_{Z}\right)$ is feasible, since $Y$ is compact set, then $u_{Z}$ is finite. If $\left(U_{\bar{Z}}\right)$ is infeasible, then $u_{\bar{Z}}=-\infty<u_{Z}$. Thus, in the following, we assume that $\left(U_{\bar{Z}}\right)$ is feasible, then $u_{\bar{Z}}$ is finite, and there exists a point $\bar{y} \in Y$ such that $\bar{L}_{i} \leq \sum_{j=1}^{n} e_{i j} \bar{y}_{j}+f_{i}$, $i=1, \ldots, p$. And

$$
u_{\bar{Z}}=\sum_{i=1}^{p}\left(\sum_{T_{i}^{+}} \bar{U}_{i} c_{i j} \bar{y}_{j}+\sum_{T_{i}^{-}} \bar{L}_{i} c_{i j} \bar{y}_{j}\right)+\sum_{D_{i}^{+}} \bar{U}_{i} d_{i}+\sum_{D_{i}^{-}} \bar{L}_{i} d_{i} .
$$

By $\bar{Z} \subseteq Z$, we have $0<L_{i} \leq \bar{L}_{i} \leq \bar{U}_{i} \leq U_{i}, i=1, \ldots$, p. It follows that $\bar{y}$ is a feasible solution of problem $\left(U_{Z}\right)$ and

$$
u_{\bar{Z}} \leq \sum_{i=1}^{p}\left(\sum_{T_{i}^{+}} U_{i} c_{i j} \bar{y}_{j}+\sum_{T_{i}^{-}} L_{i} c_{i j} \bar{y}_{j}\right)+\sum_{D_{i}^{+}} U_{i} d_{i}+\sum_{D_{i}^{-}} L_{i} d_{i} .
$$

Since $\bar{y}$ is a feasible solution of $\left(U_{Z}\right)$, we have

$$
u_{Z} \geq \sum_{i=1}^{p}\left(\sum_{T_{i}^{+}} U_{i} c_{i j} \bar{y}_{j}+\sum_{T_{i}^{+}} L_{i} c_{i j} \bar{y}_{j}\right)+\sum_{D_{i}^{+}} U_{i} d_{i}+\sum_{D_{i}^{-}} L_{i} d_{i} .
$$

By the above discussions, we have $u_{Z} \geq u_{\bar{Z}}$, and the proof is completed.

By Theorem $1,\left(U_{Z}\right)$ can provide the valid upper bound for $\left(\mathrm{EQ}_{Z}\right)$.

\section{Algorithm and Its Convergence}

In this section, we shall present a branch-and-bound algorithm for solving (EQ). The critical operation in guaranteeing the global convergence of the proposed algorithm is the selection of a suitable branching technique. In this paper, we choose a simple bisection method. For any selected rectangle $\widehat{Z}=\left\{z \in R^{p} \mid \widehat{L}_{i} \leq z_{i} \leq \widehat{U}_{i}, i=1, \ldots, p\right\} \subseteq Z^{0}$, this branching rule is given as follows:

(1) Let $\widehat{U}_{k}-\widehat{L}_{k}=\max \left\{\widehat{U}_{i}-\widehat{L}_{i} \mid i=1, \ldots, p\right\}$.

(2) Let

$$
\begin{aligned}
\widehat{Z}_{1} & =\left\{z \in R^{p} \mid \widehat{L}_{i} \leq z_{i} \leq \widehat{U}_{i}, i=1, \ldots, p, i \neq k ; \widehat{L}_{k}\right. \\
& \left.\leq z_{k} \leq \frac{1}{2}\left(\widehat{L}_{k}+\widehat{U}_{k}\right)\right\}, \\
\widehat{Z}_{2} & =\left\{z \in R^{p} \mid \widehat{L}_{i} \leq z_{i} \leq \widehat{U}_{i}, i=1, \ldots, p, i\right. \\
& \left.\neq k ; \frac{1}{2}\left(\widehat{L}_{k}+\widehat{U}_{k}\right) \leq z_{k} \leq \widehat{U}_{k}\right\} .
\end{aligned}
$$

3.1. New Region Reduction Operation. For any rectangle $Z^{k} \subseteq$ $Z^{0}$, during the process of iteration, we want to recognize whether or not $Z^{k}$ contains a global optimal solution of (EQ). The proposed new reduction operation aims at replacing the rectangle $Z^{k}=\left[L^{k}, U^{k}\right]$ with a smaller rectangle $Z^{\prime}=\left[L^{\prime}, U^{\prime}\right]$ without deleting any global optimal solution of (EQ).

Without loss of generality, we can assume that $L B^{k}$ is a known lower bound of the optimal value for $\mathrm{EQ}\left(Z^{0}\right)$ and that $v(Z)$ is the maximum value of $g(y, z)$ over $Z$ and $Y$, and let

$$
\begin{aligned}
l_{i}^{0} & =\min _{y \in Y}\left(\sum_{j=1}^{n} c_{i j} y_{j}+d_{i}\right), \quad i=1,2, \ldots, p, \\
u_{i}^{0} & =\max _{y \in Y}\left(\sum_{j=1}^{n} c_{i j} y_{j}+d_{i}\right), \quad i=1,2, \ldots, p, \\
R U B^{k} & =\sum_{i=1}^{p} u_{i}^{0} U_{i}^{k},
\end{aligned}
$$$$
\rho_{i}^{k}=\frac{L B^{k}-R U B^{k}+u_{i}^{0} U_{i}^{k}}{u_{i}^{0}}, \quad i=1,2, \ldots, p .
$$

Theorem 2. For any subrectangle $Z^{k}=\left(Z_{i}^{k}\right)_{p \times 1}=\left[L_{i}^{k}\right.$, $\left.U_{i}^{k}\right]_{p \times 1} \subseteq Z^{0}$, the following conclusions hold:

(i) If $R U B^{k}<L B^{k}$, then there is no global optimal solution of $E Q\left(Z^{0}\right)$ over $Z^{k}$. 
(ii) If $R U B^{k} \geq L B^{k}$, then, for each $s \in\{1,2, \ldots, p\}$, there is no global optimal solution of the $E Q\left(Z^{0}\right)$ over $\underline{Z}^{k}$, where

$$
\begin{aligned}
& \underline{Z}^{k}=\left(\underline{Z}_{i}^{k}\right)_{p \times 1} \subseteq Z^{0} \\
& \text { with } \underline{Z}_{i}^{k}= \begin{cases}Z_{i}^{k}, & i \neq s, i=1,2, \ldots, p, \\
{\left[L_{i}^{k}, \rho_{i}^{k}\right) \cap Z_{i}^{k}} & i=s \in\{1,2, \ldots, p\} .\end{cases}
\end{aligned}
$$

Proof. (i) If $R U B^{k}<L B^{k}$, then we have

$$
\begin{aligned}
v\left(Z^{k}\right) & =\max _{z \in Z^{k}, y \in Y} \sum_{i=1}^{p}\left(\sum_{j=1}^{n} c_{i j} y_{j}+d_{i}\right) z_{i} \\
& \leq \sum_{i=1}^{p} \max _{z \in Z^{k}, y \in Y}\left[\left(\sum_{j=1}^{n} c_{i j} y_{j}+d_{i}\right) z_{i}\right]=\sum_{i=1}^{p} u_{i}^{0} U_{i}^{k} \\
& =R U B^{k}<L B^{k} .
\end{aligned}
$$

Therefore, there exists no global optimal solution of the problem $\mathrm{EQ}\left(Z^{0}\right)$ over $Z^{k}$.

(ii) If $R U B^{\mathrm{k}} \geq L B^{k}$, then we have the following conclusions.

For each $s \in\{1,2, \ldots, p\}$, for any $y \in Y$ and $z \in Z^{k}$, and for each $i=1,2, \ldots, p$, since

$$
\begin{aligned}
& 0 \leq l_{i}^{0} \leq \sum_{j=1}^{n} c_{i j} y_{j}+d_{i} \leq u_{i}^{0}, \\
& 0 \leq L_{i}^{k} \leq z_{i} \leq U_{i}^{k}, \quad i \neq s, \\
& 0 \leq L_{s}^{k} \leq z_{s} \leq \rho_{s}^{k}, \quad i=s,
\end{aligned}
$$

we have

$$
\begin{aligned}
v\left(\underline{Z}^{k}\right)= & \max _{z \in \underline{Z}^{k}, y \in Y}\left[\sum_{i=1}^{p}\left(\sum_{j=1}^{n} c_{i j} y_{j}+d_{i}\right) z_{i}\right] \\
\leq & \max _{z \in \underline{Z}^{k}, y \in Y}\left[\sum_{i=1, i \neq s}^{p}\left(\sum_{j=1}^{n} c_{i j} y_{j}+d_{i}\right) z_{i}\right] \\
& +\max _{z \in \underline{Z}^{k}, y \in Y}\left[\left(\sum_{j=1}^{n} c_{s j} y_{j}+d_{s}\right) z_{s}\right] \\
< & \sum_{i=1, i \neq s}^{p} u_{i}^{0} U_{i}^{k}+u_{s}^{0} \rho_{s}^{k} \\
= & R U B^{k}-u_{s}^{0} U_{s}^{k}+L B^{k}-\left(R U B^{k}-u_{s}^{0} U_{s}^{k}\right) \\
= & L B^{k} .
\end{aligned}
$$

Therefore, there exists no global optimization solution of $\mathrm{EQ}\left(Z^{0}\right)$ over $\underline{Z}^{k}$.
By Theorem 2, we can construct a new reduction operation to cut away a part of region in which the global optimal solution of (EQ) does not exist. Assuming that a subrectangle

$$
Z^{k}=\left(Z_{i}^{k}\right)_{p \times 1} \subseteq Z^{0} \quad \text { with } Z_{i}^{k}=\left[L_{i}^{k}, U_{i}^{k}\right]
$$

will be compressed, then, according to Theorem 2, the original rectangle $Z^{k}$ should be replaced with a new subrectangle:

$$
Z^{\prime}=\left(Z_{i}^{\prime}\right)_{p \times 1}
$$

$$
\text { with } Z_{i}^{\prime}=\left[\rho_{i}^{k}, U_{i}^{k}\right] \cap Z_{i}^{k}, i \in\{1,2, \ldots, p\} \text {. }
$$

Let $U B\left(Z^{k}\right)$ be the optimal value of $\left(U_{Z}\right)$ in $Z^{k}$; the basic steps of the proposed algorithm are summarized as follows.

\subsection{Algorithm Steps}

Step 0. Initialize the iteration counter $k=0$, the active node set $\Omega_{0}=\left\{Z^{0}\right\}$, the lower bound $L B_{0}=-\infty$, and the feasible points set $F=\emptyset$.

Solve $\left(U_{Z^{0}}\right)$; obtain $u_{Z^{0}}$ and $y\left(Z^{0}\right)$. Set $U B_{0}=U B\left(Z^{0}\right)=$ $u_{Z^{0}}, y^{c}=y\left(Z^{0}\right)$, and set $z_{i}^{c}=\sum_{j=1}^{n} e_{i j} y_{j}^{c}+f_{i}(i=1,2, \ldots, p)$, $L B_{0}=g\left(y^{c}, z^{c}\right)$. Update $F=F \cup\left\{\left(y^{c}, z^{c}\right)\right\}$.

If $U B_{0}-L B_{0} \leq \epsilon$, then the algorithm stops with $\left(y^{c}, z^{c}\right)$ and $y^{c}$ as global optimal solutions of (EQ) and (GAMP), respectively. Otherwise, proceed to next step.

Step 1. Use the proposed reduction operation to compress the investigated rectangle $Z^{k}$, and still represent remaining part of the rectangle as $Z^{k}$.

Step 2. Subdivide $Z^{k}$ into two subrectangles; denote by $\Theta_{k}$ its partitioning set.

For each $Z \in \Theta_{k}$, solve $\left(U_{Z}\right)$ to obtain $U B(Z)=u_{Z}$ and $y(Z)$. If $U B(Z)<L B_{k}$, then remove the subrectangle $Z$ from $\Theta_{k}$, that is, $\Theta_{k}:=\Theta_{k} \backslash Z$, and skip to next element of $\Theta_{k}$.

If $\Theta_{k}=\emptyset$, set $L B_{k+1}=L B_{k}$, and go to Step 3. Otherwise, set $z_{i}^{c}=z_{i}(Z)=\sum_{j=1}^{n} e_{i j} y_{j}(Z)+f_{i}, i=1,2, \ldots, p$, update $F=F \cup\{(y(Z), z(Z))\}$, set $L B_{k+1}=\max _{(y, z) \in F} g(y, z)$, and denote by $\left(y^{c}, z^{c}\right)=\operatorname{argmax}_{(y, z) \in F} g(y, z)$ the best known feasible point.

Step 3. Denote by $\Omega_{k+1}=\left(\Omega_{k} \backslash Z^{k}\right) \cup \Theta_{k}$ the remaining partition set. We can obtain a new upper bound $U B_{k}:=$ $\inf _{Z \in \Omega_{k+1}} U B(Z)$.

Step 4. Fathom any nonimproving nodes by setting $\Omega_{k+1}=$ $\Omega_{k} \backslash\left\{Z: U B(Z)-L B \leq \epsilon, Z \in \Omega_{k}\right\}$. If $\Omega_{k+1}=\emptyset$, then stop: $L B$ is the optimal value of (EQ), and $y^{c}$ is a global $\epsilon$-optimal solutions for (GAMP). Otherwise, set $k=k+1$ and return to Step 1.

3.3. Convergence of the Algorithm. In this subsection, the convergence of the algorithm is given.

Theorem 3. If the algorithm is finite, upon termination, we can obtain the $\epsilon$-global optimal solutions for the problems (EQ) and 
(GAMP), respectively. If the algorithm is infinite, for each $k \geq$ 0 , denote by $y^{k}$ the incumbent solution of (GAMP), which is found at the end of iteration $k$. Then, $\lim _{k \rightarrow \infty} y^{k}=y^{*}$ will a global optimal solution for (GAMP).

Proof. If the algorithm is finite, then the algorithm terminates at iteration $k, k \geq 0$. By the termination condition of the algorithm and the updating method of the upper bound, it can follow easily that the conclusion is obvious.

If the algorithm is infinite, then, it generates a sequence of incumbent solutions for problem (EQ), which may be denoted by $\left\{\left(y^{k}, z^{k}\right)\right\}$. For each $k \geq 1,\left(y^{k}, z^{k}\right)$ is found by solving problem $\left(U_{Z^{k}}\right)$, for some rectangle $Z^{k} \subseteq Z^{0}$, for an optimal solution $y^{k} \in Y$, and setting $z_{i}^{k}=\sum_{j=1}^{n} e_{i j} y_{j}^{k}+f_{i}$, $i=1, \ldots, p$. Therefore, the sequence $\left\{y^{k}\right\}$ consists of feasible solutions for problem (GAMP). Let $y^{*}$ be an accumulation point of $\left\{y^{k}\right\}$, and without loss of generality we assume that $\lim _{k \rightarrow \infty} y^{k}=y^{*}$. Then, since $Y$ is a compact set, $y^{*} \in Y$. Furthermore, since $\left\{y^{k}\right\}$ is infinite, we may assume without loss of generality that, for each $k, Z^{k+1} \subseteq Z^{k}$. Since the rectangles $Z^{k}, k \geq 1$, are formed by rectangular bisection, this implies that, for some point $z^{*} \in R^{p}$, we have

$$
\lim _{k \rightarrow \infty} Z^{k}=\bigcap_{k} Z^{k}=\left\{z^{*}\right\} .
$$

Let $Z^{*}=\left\{z^{*}\right\}$ and, for each $k$, let $Z^{k}$ be given by

$$
Z^{k}=\left\{z \in R^{p} \mid L^{k} \leq z \leq U^{k}\right\} .
$$

For each $k$, from Step 2 of the algorithm,

$$
U B\left(Z^{k}\right)=u_{Z^{k}} .
$$

Since $Z^{k+1} \subset Z^{k} \subseteq Z^{0}$, for each $k$, by Theorem 1 and Step 4, this implies that $\left\{U B\left(Z^{k}\right)\right\}$ is a nonincreasing sequence bounded by $v$. Therefore, $\lim _{k \rightarrow \infty} U B\left(Z^{k}\right)$ is a finite number and satisfies

$$
\lim _{k \rightarrow \infty} U B\left(Z^{k}\right) \geq v
$$

For each $k$, from Step $1, U B\left(Z^{k}\right)$ is equal to the optimal value of problem $\left(U_{Z^{k}}\right)$ given by

$$
\begin{aligned}
\max \quad \sum_{i=1}^{p}\left(\sum_{T_{i}^{+}} U_{i}^{k} c_{i j} y_{j}+\sum_{T_{i}^{-}} L_{i}^{k} c_{i j} y_{j}\right)+\sum_{D_{i}^{+}} U_{i}^{k} d_{i} \\
+\sum_{D_{i}^{-}} L_{i}^{k} d_{i} \\
\text { s.t. } \quad L_{i}^{k}-\left(\sum_{j=1}^{n} e_{i j} y_{j}+f_{i}\right) \leq 0, \quad i=1, \ldots, p, \\
y \in Y,
\end{aligned}
$$

and $y^{k}$ is an optimal solution for this problem. By the branching technique, we have

$$
\lim _{k \rightarrow \infty} L^{k}=\lim _{k \rightarrow \infty} U^{k}=\left\{z^{*}\right\}=Z^{*} .
$$

Since $\lim _{k \rightarrow \infty} y^{k}=y^{*}, L_{i}^{k} \leq \sum_{j=1}^{n} e_{i j} y_{j}^{k}+f_{i} \leq U_{i}^{k}$ and the continuity of $\left(\sum_{j=1}^{n} e_{i j} y_{j}+f_{i}\right)$, for each $i=1, \ldots, p$, $\sum_{j=1}^{n} e_{i j} y_{j}^{*}+f_{i}=z_{i}^{*}$. This implies that $\left(y^{*}, z^{*}\right)$ is a feasible solution for (EQ). Therefore, $g\left(y^{*}, z^{*}\right) \leq v$. Combing (20), we obtain that

$$
g\left(y^{*}, z^{*}\right) \leq v \leq \lim _{k \rightarrow \infty} U B\left(Z^{k}\right)
$$

since

$$
\begin{aligned}
& \lim _{k \rightarrow \infty} U B\left(Z^{k}\right)=\lim _{k \rightarrow \infty}\left(\sum_{i=1}^{p}\left(\sum_{T_{i}^{+}} U_{i}^{k} c_{i j} y_{j}^{k}+\sum_{T_{i}^{-}} L_{i}^{k} c_{i j} y_{j}^{k}\right)\right. \\
& \left.+\sum_{D_{i}^{+}} U_{i}^{k} d_{i}+\sum_{D_{i}^{-}} L_{i}^{k} d_{i}\right)=\sum_{i=1}^{p} z_{i}^{*}\left(\sum_{j=1}^{n} c_{i j} y_{j}^{*}+d_{i}\right) \\
& =g\left(y^{*}, z^{*}\right) .
\end{aligned}
$$

From (22) and (23), since $U B\left(Z^{k}\right)=U B_{k}$, we have $\lim _{k \rightarrow \infty} U B_{k}=v=g\left(y^{*}, z^{*}\right)$. Therefore, $\left(y^{*}, z^{*}\right)$ is a global optimal solution for (EQ). By Theorem 1, this implies that $y^{*}$ is a global optimal solution for (GAMP). The proof is complete.

\section{Numerical Experiments}

To verify the performance of the proposed algorithm, some test problems are implemented on an Intel(R) Core(TM)2 Duo CPU (1.58 GHZ) microcomputer. The algorithm is coded in $\mathrm{C}++$, and each linear programs problem is solved by simplex method in our experiments. These test problems are given in the following, and their numerical results are listed in Tables 1 and 2.

Example 1 (see [14]). One has

$$
\begin{array}{ll}
\max \quad\left(3 y_{1}+5 y_{2}+3 y_{3}+50\right) \\
\quad \times\left(3 y_{1}+4 y_{2}+5 y_{3}+50\right)+\left(3 y_{1}+4 y_{2}+50\right) \\
\quad \times\left(4 y_{1}+3 y_{2}+2 y_{3}+50\right) \\
\quad+\left(4 y_{1}+2 y_{2}+4 y_{3}+50\right) \\
\quad \times\left(5 y_{1}+4 y_{2}+3 y_{3}+50\right) \\
\text { s.t. } \quad 6 y_{1}+3 y_{2}+3 y_{3} \leq 10 \\
& 10 y_{1}+3 y_{2}+8 y_{3} \leq 10 \\
& y_{1}, y_{2}, y_{3} \geq 0 .
\end{array}
$$


TABLE 1: Computational results of Examples 1 and 2.

\begin{tabular}{|c|c|c|c|c|c|c|}
\hline Example & Ref. & Optimal solution & Optimal value & Iteration & $\epsilon$ & Time(s) \\
\hline \multirow{2}{*}{ Example 1} & This paper & $(0,3.33333,0)$ & 11611.1 & 2 & $10^{-6}$ & 0.0065 \\
\hline & {$[14]$} & $(0,3.33333,0)$ & 11611.1 & 2 & $10^{-6}$ & 0.0074 \\
\hline \multirow{2}{*}{ Example 2} & This paper & $(0,0.625,1.875)$ & 14214.8 & 1 & $10^{-6}$ & 0.0198 \\
\hline & [14] & $(0,0.625,1.875)$ & 14214.8 & 2 & $10^{-6}$ & 0.0228 \\
\hline
\end{tabular}

TABLE 2: Numerical results for Example 3.

\begin{tabular}{lcc}
\hline$n$ & $p$ & Time(s) \\
\hline 10 & 10 & 0.0285 \\
20 & 10 & 0.0192 \\
30 & 10 & 0.0279 \\
30 & 30 & 0.0590 \\
30 & 40 & 0.1102 \\
30 & 50 & 0.0945 \\
35 & 50 & 0.1283 \\
40 & 50 & 0.2423 \\
50 & 50 & 0.2899 \\
\hline
\end{tabular}

Example 2 (see [14]). One has

$$
\begin{array}{ll}
\max \quad\left(4 y_{1}+3 y_{2}+3 y_{3}+50\right) \times\left(3 y_{2}+3 y_{3}+50\right) \\
+ & \left(3 y_{1}+4 y_{3}+50\right) \times\left(4 y_{1}+4 y_{2}+5 y_{3}+50\right) \\
& +\left(y_{1}+2 y_{2}+5 y_{3}+50\right) \\
& \times\left(y_{1}+5 y_{2}+5 y_{3}+50\right) \\
& +\left(y_{1}+2 y_{2}+4 y_{3}+50\right) \times\left(5 y_{2}+4 y_{3}+50\right) \\
\text { s.t. } \quad 2 y_{1} & +y_{2}+5 y_{3} \leq 10 \\
& y_{1}+6 y_{2}+3 y_{3} \leq 10 \\
5 & y_{1}+9 y_{2}+2 y_{3} \leq 10 \\
9 & y_{1}+7 y_{2}+3 y_{3} \leq 10 \\
& y_{1}, y_{2}, y_{3} \geq 0 .
\end{array}
$$

In Table 1, the notations have been used for column headers: iteration, number of iterations; time: execution time(s) of the algorithm in seconds.

Example 3. One has

$$
\begin{aligned}
\max & \sum_{i=1}^{p}\left(\sum_{j=1}^{n} c_{i j} y_{j}+d_{i}\right)\left(\sum_{j=1}^{n} e_{i j} y_{j}+f_{i}\right) \\
\text { s.t. } & 0 \leq y_{j} \leq \gamma_{j}, \quad j=1, \ldots, n,
\end{aligned}
$$

where $c_{i j}, e_{i j}, d_{i}, f_{i}, i=1, \ldots, p, j=0,1, \ldots, n$ are randomly generated between 0 and $1 ; \gamma_{j}(j=1, \ldots, n)$ were randomly generated between 0 and 16 .
Numerical results for Example 3 are given in Table 2, where the convergence tolerance is set to $\epsilon=10^{-6}$.

From Tables 1 and 2, it is seen that the proposed algorithm in this paper can be used to globally solve the problem (GAMP) with the effectiveness and robustness.

\section{Concluding Remarks}

In this article, an out space accelerating branch-and-bound algorithm is presented for globally solving the generalized affine multiplicative programs problem (GAMP). First of all, we transform the (GAMP) into an equivalent problem (EQ). By utilizing the new linear relaxation technique, we systematically convert (EQ) into a series of linear relaxation programs problems, which can infinitely approximate the global optimal solution of (EQ) by a successive partition. To improve the computational efficiency of the algorithm, we introduce an out space reduction operation, which offers a theoretical possibility of reducing a large part of the investigated out space region and which can be seen as an accelerating device for improving the convergent speed of the proposed algorithm. By combining the established linear relaxed programs problem with the new reduction operation in a branch-and-bound framework, we design an out space accelerating algorithm for effectively solving (GAMP). By subsequently dividing the initial rectangle and subsequently solving a series of linear relaxed programs problems, the presented algorithm is convergent to the global minimum of (GAMP). Compared with the known algorithms, numerical results demonstrate that the proposed algorithm has higher computational efficiency.

\section{Conflicts of Interest}

The authors declare that they have no conflicts of interest.

\section{Acknowledgments}

This paper is supported by the Basic and Advanced Technology Research Project of Henan Province (152300410097), the Key Scientific Research Project of Universities in Henan Province (17B413002, 16A110014, and 17A110021), the Major Scientific Research Projects of Henan Institute of Science and Technology (2015ZD07), the National Natural Science Foundation of Henan Province (152300410097), and Science and Technology Project of Henan Province (162102210294). 


\section{References}

[1] H. Konno, P. T. Thach, and H. Tuy, Optimization on Low Rank Nonconvex Structures, vol. 15 of Nonconvex Optimization and its Applications, Kluwer Academic Publishers, Dordrecht, The Netherlands, 1997.

[2] J. M. Henderson and R. E. Quandt, Microeconomic Theorey, McGraw-Hill, New York, NY, USA, 2nd edition, 1971.

[3] K. Swarup, "Programming with indefinite quadratic function with linear constraints," Cahier du Coutre d'Etudes de Recherche Operationelle, vol. 8, pp. 223-234, 1966.

[4] H.-M. Li and K.-C. Zhang, "A decomposition algorithm for solving large-scale quadratic programming problems," Applied Mathematics and Computation, vol. 173, no. 1, pp. 394-403, 2006.

[5] H. Wu and K. Zhang, "A new accelerating method for global non-convex quadratic optimization with non-convex quadratic constraints," Applied Mathematics and Computation, vol. 197, no. 2, pp. 810-818, 2008.

[6] S.-T. Liu and R.-T. Wang, "A numerical solution method to interval quadratic programming," Applied Mathematics and Computation, vol. 189, no. 2, pp. 1274-1281, 2007.

[7] P. Shen and M. Gu, "A duality-bounds algorithm for non-convex quadratic programs with additional multiplicative constraints," Applied Mathematics and Computation, vol. 198, no. 1, pp. 1-11, 2008.

[8] P. Shen, Y. Duan, and Y. Ma, "A robust solution approach for nonconvex quadratic programs with additional multiplicative constraints," Applied Mathematics and Computation, vol. 201, no. 1-2, pp. 514-526, 2008.

[9] H. Konno and K. Fukaishi, "A branch and bound algorithm for solving low rank linear multiplicative and fractional programming problems," Journal of Global Optimization, vol. 18, no. 3, pp. 283-299, 2000.

[10] H.-S. Ryoo and N. V. Sahinidis, "Global optimization of multiplicative programs," Journal of Global Optimization, vol. 26, no. 4, pp. 387-418, 2003.

[11] C. Xue, H. Jiao et al., "An approximate algorithm for solving generalized linear multiplicative programming," Journal of Henan Normal University (Natural Science), vol. 36, no. 5, pp. 13-15, 2008.

[12] H. Tuy and N. Duc, "Reverse polyblock approximation for generalized multiplicative/fractional programming," Vietnam Journal of Mathematics, vol. 31, no. 4, pp. 391-402, 2003.

[13] S. Schaible and C. Sodini, "Finite algorithm for generalized linear multiplicative programming," Journal of Optimization Theory and Applications, vol. 87, no. 2, pp. 441-455, 1995.

[14] H. Jiao, S. Liu, and Y. Chen, "Global optimization algorithm for a generalized linear multiplicative programming," Journal of Applied Mathematics and Computing, vol. 40, no. 1-2, pp. 551568, 2012.

[15] H. P. Benson and G. M. Boger, "Outcome-space cutting-plane algorithm for linear multiplicative programming," Journal of Optimization Theory and Applications, vol. 104, no. 2, pp. 301$322,2000$.

[16] X. J. Liu, T. Umegaki, and Y. Yamamoto, "Heuristic methods for linear multiplicative programming," Journal of Global Optimization, vol. 15, no. 4, pp. 433-447, 1999.

[17] N. Thi and H. Tuy, "A unified monotonic approach to generalized linear fractional programming," Journal of Global Optimization, vol. 26, no. 3, pp. 229-259, 2003.
[18] Y. Chen and H. Jiao, "A nonisolated optimal solution of general linear multiplicative programming problems," Computers and Operations Research, vol. 36, no. 9, pp. 2573-2579, 2009.

[19] C.-F. Wang, S.-Y. Liu, and P.-P. Shen, "Global minimization of a generalized linear multiplicative programming," Applied Mathematical Modelling, vol. 36, no. 6, pp. 2446-2451, 2012.

[20] H.-W. Jiao, S.-Y. Liu, and Y.-F. Zhao, "Effective algorithm for solving the generalized linear multiplicative problem with generalized polynomial constraints," Applied Mathematical Modelling, vol. 39, no. 23-24, pp. 7568-7582, 2015. 


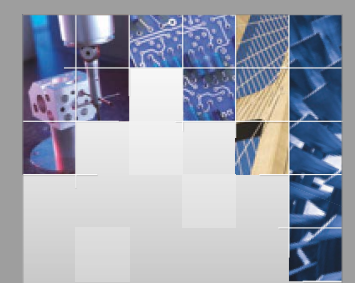

\section{Enfincering}
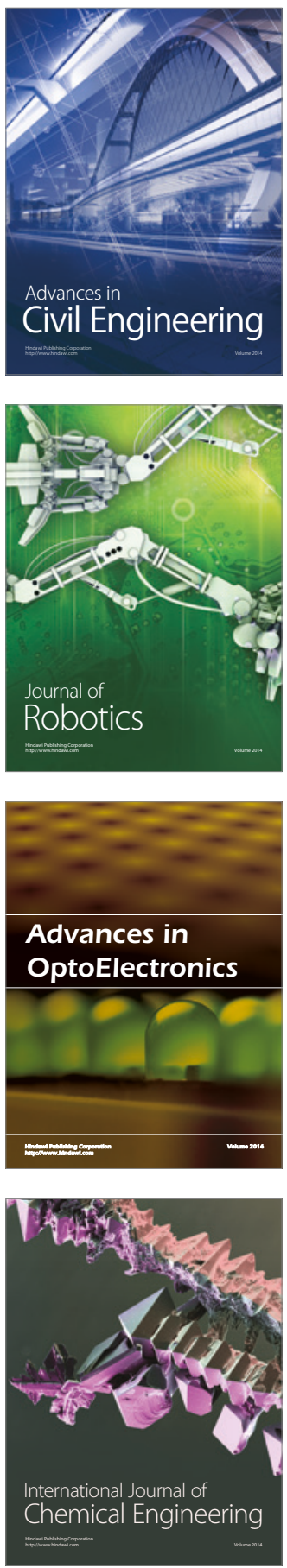

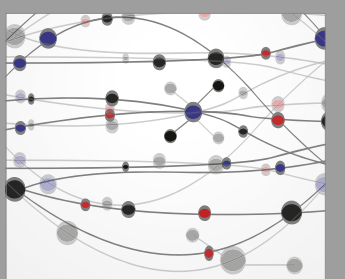

The Scientific World Journal

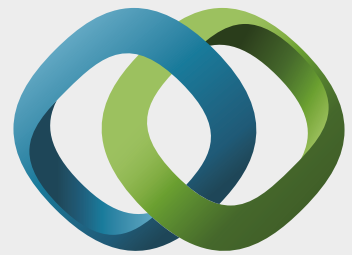

\section{Hindawi}

Submit your manuscripts at

https://www.hindawi.com
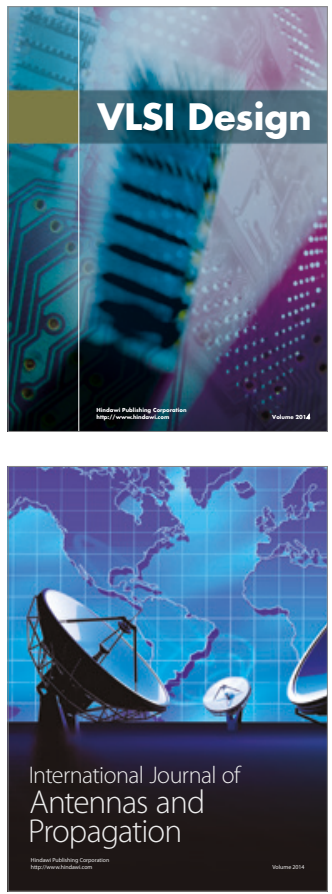

\section{Rotating}

Machinery
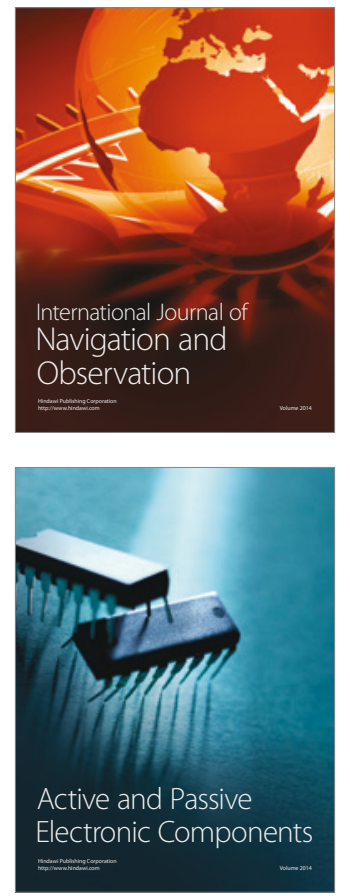
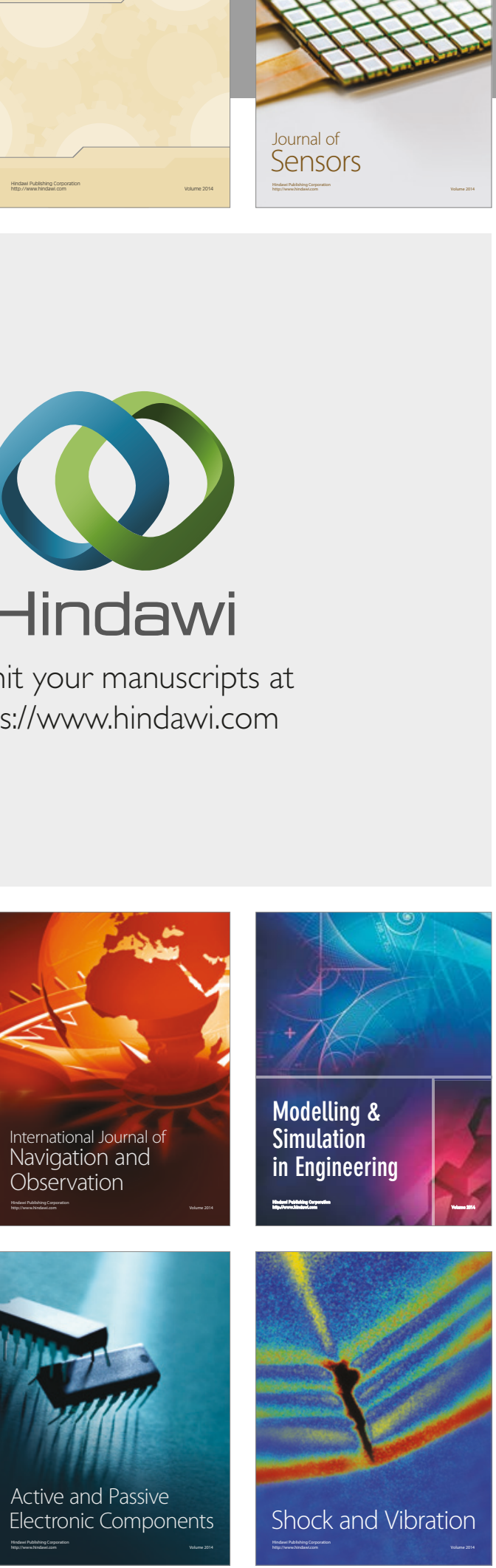
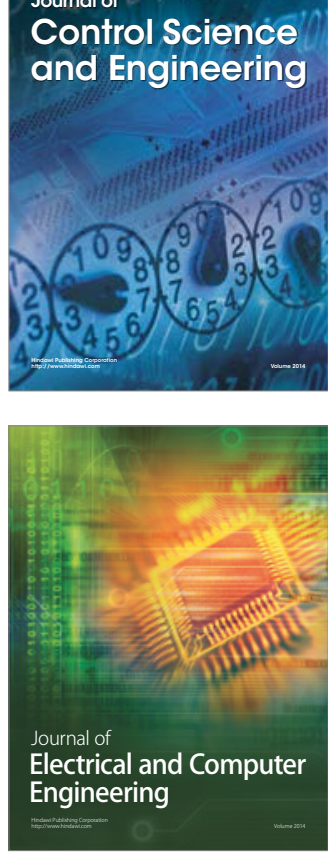

Distributed

Journal of

Control Science

and Engineering


Sensor Networks 